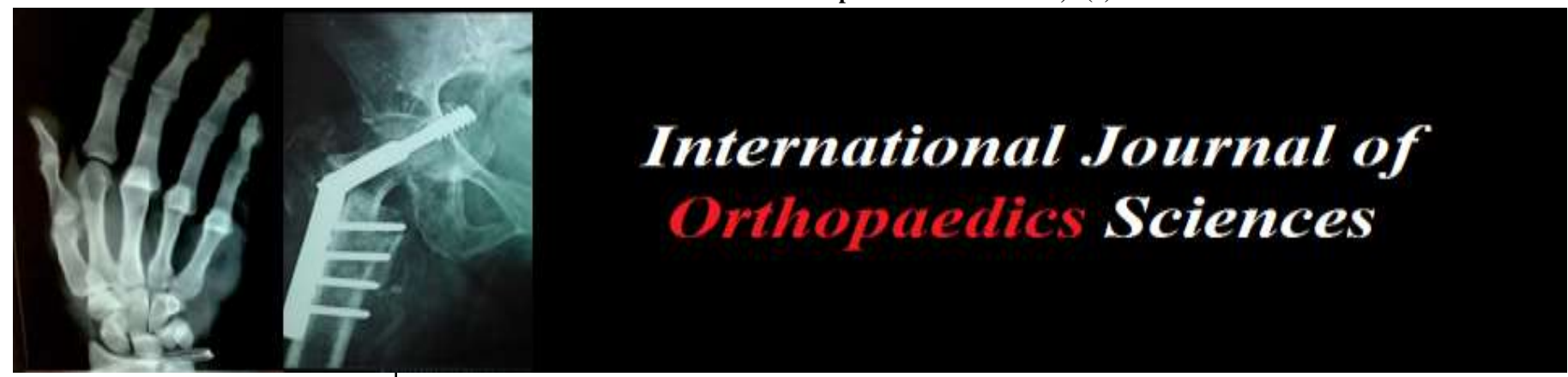

E-ISSN: 2395-1958

P-ISSN: 2706-6630

IJOS 2020; 6(4): 689-692

(C) 2020 IJOS

www.orthopaper.com

Received: 05-08-2020

Accepted: 11-09-2020

Dr. Avinash Kuma

Senior Resident, Department of

Orthopaedics, Mata Gujari

Memorial Medical College and

Lions Seva Kendra Hospital,

Kishanganj, Bihar, India

Dr. Anubhav Jain

Senior Resident, Department of

Orthopaedics, Mata Gujari

Memorial Medical College and

Lions Seva Kendra Hospital

Kishanganj, Bihar, India

Dr. Saurabh Kumar

PG Resident, Department of

Orthopaedics, Mata Gujari

Memorial Medical College and

Lions Seva Kendra Hospital,

Kishanganj, Bihar, India

Dr. Rahul Raj

PG Resident, Department of Orthopaedics, Mata Gujari

Memorial Medical College and

Lions Seva Kendra Hospital,

Kishanganj, Bihar, India
Corresponding Author: Dr. Anubhav Jain

Senior Resident, Department of Orthopaedics, Mata Gujari

Memorial Medical College and

Lions Seva Kendra Hospital,

Kishanganj, Bihar, India

\section{Study on clinical features and outcome of Monteggia fracture dislocation in adults}

\author{
Dr. Avinash Kumar, Dr. Anubhav Jain, Dr. Saurabh Kumar and Dr. \\ Rahul Raj
}

DOI: https://doi.org/10.22271/ortho.2020.v6.i4j.2407

\section{Abstract}

Background: Monteggia fracture comprise of 5\% to $10 \%$ of all fractures of forearm. The rates of complication and re-dislocation are high following the surgery of these lesions.

Methods: The present observational study included 29 cases of Monteggia fracture. Clinical details, details of management and outcome were noted.

Results: Majority of the subjects were in the age group of 21-30 years. Type 1 was the most common injury seen $(75.9 \%)$ according to the Bado's classification. In $6.9 \%$, it did not unite even after six months. Excellent outcome was seen in $65.5 \%$ cases while poor result was obtained in $6.9 \%$ cases. Superficial infection was seen in three cases and non-union in two cases.

Conclusion: Early treatment of Monteggia fracture-dislocation is helpful in preventing complications.

Keywords: Clinical features, Monteggia fracture dislocation, observational study, outcome

\section{Introduction}

Monteggia fracture are severe injuries of the forearm which need surgical intervention. These comprise of $5 \%$ to $10 \%$ of all fractures of forearm ${ }^{[1]}$. Persistence of radial head dislocation causes increasing valgus deformity of the elbow which leads to damage to ulnar or radial nerve. The range of motion is restricted because of obstruction by the radial head. Long standing Monteggia fracture-dislocation can result in secondary degenerative arthritis ${ }^{[2]}$.

Hence, early treatment of Monteggia fracture-dislocation is important to prevent complications. The treatment of neglected Monteggia fracture dislocation is challenging and a number of surgical techniques have been introduced. The rates of complication and redislocation are high following the surgery of these lesions ${ }^{[3]}$.

Various articles have discussed about the treatment options and complications of these fractures ${ }^{[1,2,4,5]}$. Still, there is no unanimous opinion. Also, studies regarding this issue have not been conducted in this area. Hence, the present study was proposed.

Aims \& objectives: The present study was conducted to assess the outcome and complications of Monteggia fracture-dislocation in patients admitted at a medical college hospital.

\section{Material and methods}

- Study design: Hospital- based observational study.

- Study place: The present study was conducted at the department of Orthopaedics, Mata Gujari Memorial Medical College and Lions Seva Kendra Hospital, Kishanganj.

- Period of study: The present study was conducted between August 2017 to March 2019.

- Study population: The study population included adult patients suffering from forearm fracture.

- Inclusion criteria: The patients above 18 years of age suffering from forearm fracture and diagnosed to be suffering from Monteggia fracture dislocation were included in the present study.

- Exclusion criteria: Open fracture, polytrauma, neurovascular injury and pathological fractures were excluded.

- Sampling: All the patients reporting with Monteggia fracture dislocation during the study period were included. A total of 29 patients were studied. 
- Study tools: Pre-tested proforma was used for data collection. It included questions related to demographic profile, clinical diagnosis, findings of radiological examination, details of surgical procedure and outcome.

- Data collection procedure: The study subjects were recruited from the Orthopaedics department of the institute. Details of the patients including demographic details, history of illness, clinical features, findings of laboratory and radiological examinations were noted. Details of management and outcome were also noted. The patients were followed up for six months.

- Data analysis: Data entry was entered in Microsoft Excel 2010 and analysed using Statistical Package for Social Sciences (SPSS) v 20.0. Data were summarized as mean and SD or frequency and percentage. Appropriate statistical tests were done. p-value $<0.05$ was considered to be statistically significant.

- Ethical consideration \& permission: Permission was obtained from Institutional Ethics Committee. Informed consent was taken from all the study subjects.

\section{Results}

Majority of the subjects were in the age group of 21-30 years. $58.6 \%$ of them were males. Right side was involved in $55.2 \%$. Road traffic accident was the most common cause of this injury (48.3\%). Type 1 was the most common injury seen (75.9\%) according to the Bado's classification.

Table 1: Showing demographic and clinical profile of the patients

\begin{tabular}{|c|c|c|c|}
\hline Characteristics & Values & Frequency & $\mathbf{\%}$ \\
\hline \multirow{4}{*}{ Age (in years) } & $21-30$ & 13 & 44.8 \\
\cline { 2 - 4 } & $31-40$ & 8 & 27.6 \\
\cline { 2 - 4 } & $41-50$ & 6 & 20.7 \\
\cline { 2 - 4 } & $>50$ & 2 & 6.9 \\
\hline \multirow{2}{*}{ Sex } & Male & 17 & 58.6 \\
\cline { 2 - 4 } & Female & 12 & 41.4 \\
\hline \multirow{2}{*}{ Side involved } & Right & 16 & 55.2 \\
\cline { 2 - 4 } & Left & 13 & 44.8 \\
\hline \multirow{3}{*}{ Mode of injury } & RTA & 14 & 48.3 \\
\cline { 2 - 4 } & Fall & 13 & 44.8 \\
\cline { 2 - 4 } Type of fracture according to Bado's classification & Violence & 2 & 6.9 \\
\cline { 2 - 4 } & Type 1 & 22 & 75.9 \\
\cline { 2 - 4 } & Type 2 & 5 & 17.2 \\
\cline { 2 - 4 } & Type 3 & 2 & 6.9 \\
\cline { 2 - 4 } & Type 4 & 0 & 0 \\
\hline \multirow{2}{*}{}
\end{tabular}

In $89.7 \%$ cases, radial head reduction was done while radial head excision was needed in 6.95 cases. Annular ligament was repaired in one case.

Table 2: Showing the treatment done

\begin{tabular}{|c|c|c|}
\hline Treatment done & Frequency & \% \\
\hline Radial head reduction & 26 & 89.7 \\
\hline Radial head excision & 2 & 6.9 \\
\hline Repair of annular ligament & 1 & 3.4 \\
\hline
\end{tabular}

Union was seen within four months in $82.8 \%$ cases while in $6.9 \%$, it did not unite even after six months. Excellent outcome was seen in $65.5 \%$ cases while poor result was obtained in $6.9 \%$ cases. Superficial infection was seen in three cases and non-union in two cases.

Table 3: Showing the outcome of management

\begin{tabular}{|c|c|c|c|}
\hline Characteristics & Values & Frequency & \% \\
\hline \multirow{3}{*}{ Time taken for union } & $<4$ & 24 & 82.8 \\
\cline { 2 - 4 } & $4-6$ & 3 & 10.3 \\
\cline { 2 - 4 } & $>6$ & 2 & 6.9 \\
\hline \multirow{3}{*}{ Anderson scoring } & Excellent & 19 & 65.5 \\
\cline { 2 - 4 } & Good & 6 & 20.7 \\
\cline { 2 - 4 } & Fair & 2 & 6.9 \\
\cline { 2 - 4 } & Poor & 2 & 6.9 \\
\hline
\end{tabular}

Table 4: Showing complications

\begin{tabular}{|c|c|c|}
\hline Complications & Frequency & $\boldsymbol{\%}$ \\
\hline Superficial infection & 3 & 10.3 \\
\hline Non-union & 2 & 6.9 \\
\hline
\end{tabular}

\section{Discussion}

Radial head dislocations are generally associated with fractures of proximal ulna ${ }^{[6]}$. This pattern of fracture was first described by Monteggia. Later, Perin named this fracture as Monteggia fracture dislocation. Bado classified it further into four types according to angulation of the ulna fracture and direction of the radial head dislocation ${ }^{[7]}$. These are as follows- Type I, anterior dislocation and anterior angulation; Type II, posterior dislocation and posterior angulation; Type III, lateral dislocation and lateral angulation; and Type IV, proximal third fracture of both bones and anterior dislocation of the radial head ${ }^{[8]}$.

These injuries are common in children. In adults, type II is more common (80\%) while Type 1 lesions are more common in children. Monteggia fracture dislocations contribute to $5 \%$ of forearm fractures ${ }^{[3]}$.

This fracture needs urgent treatment for better outcome. Early closed reduction of radial head and open reduction \& internal fixation of ulna using compression plate is effective in treating Monteggia fracture dislocation in adults with excellent functional outcome. Immobilization should be done in 110-120 degrees of elbow flexion and forearm in supination to prevent radial head redislocation. Spontaneous reduction of the radial head depends upon anatomic reduction of the ulna. If the annular ligament hampers the closed reduction of the radial head, an open reduction must be performed $^{[4]}$.

The present study was conducted upon 29 cases suffering from Monteggia fracture dislocation. Clinical details were obtained. The patients were managed according to fracture type and condition of the patient. They were followed up for six months to assess the outcome. The fracture was considered to be united if tenderness was absent, there was no complain and the fracture line was not visible radiologically. Delayed union was diagnosed if the fracture healed after 6 months without need for any additional procedure. Non-union was considered which did not unite after six months or that 
needed additional operation procedure. The outcome was evaluated using Anderson scoring system ${ }^{[4]}$. The result was graded as excellent / good when the union of the fracture, loss of flexion and extension at the wrist or elbow of less than 10o, and loss of pronation and supination of $<25 \%$. In a fair/ satisfactory result, there was union of fracture, loss of flexion or extension at the wrist or elbow of less than $20^{\circ}$, and loss of pronation and supination of less than 50\%. The result was scored as bad when there was union of the fracture and either loss of flexion and extension at the wrist or elbow of more than $20^{\circ}$ or loss of pronation and supination of more than $50 \%$. A non-union or unresolved chronic osteomyelitis was graded as failure.

It was seen in the present study that majority of the subjects were in the age group of $21-30$ years. $58.6 \%$ of them were males. Right side was involved in 55.2\%. Road traffic accident was the most common cause of this injury (48.3\%). Type 1 was the most common injury seen $(75.9 \%)$ according to the Bado's classification. In $89.7 \%$ cases, radial head reduction was done while radial head excision was needed in 6.95 cases. Annular ligament was repaired in one case. Union was seen within four months in $82.8 \%$ cases while in $6.9 \%$, it did not unite even after six months. Excellent outcome was seen in $65.5 \%$ cases while poor result was obtained in $6.9 \%$ cases. (Chart-1) Superficial infection was seen in three cases and non-union in two cases.

Babu et al. conducted a study on 20 cases of Monteggia fracture dislocation in adults who were treated by open reduction and internal fixation using $\mathrm{AO} 3.5 \mathrm{~mm}$ dynamic Compression Plate of ulna with closed reduction of radial head. Majority of the patients were males and middle aged. Road traffic accidents were the commonest mode of injury. Most of the cases were type-1 fracture-dislocation according to Bado's classification. Upper limb was immobilized in 110120 degrees of flexion of the elbow with forearm in supination to prevent radial head redislocation. Excellent or full range of mobility of elbow and wrist joints was present in
$65 \%$ patients, $30 \%$ showed satisfactory results, $5 \%$ had unsatisfactory result and cases of failures were not seen ${ }^{[9]}$.

Khan observed that Monteggia fracture dislocation was most commonly seen in 2nd and 3rd decade with average of 35.9 years. There was male preponderance with male to female ratio being 1.5:1. $65 \%$ cases had fracture on right side and $35 \%$ cases had left side involvement. $45 \%$ patients had road traffic accidents, $40 \%$ patients had fall and $15 \%$ patients with assault. $80 \%$ of the cases were of type- 1 Bado's classification, $15 \%$ of type-3, $5 \%$ type- 4 and none of type- 2 fractures. Using Anderson et al. scoring system, 65\% cases had excellent results, $30 \%$ with satisfactory results and 5\% had failure. Three patients had superficial infection which resolved on treatment. One patient had non-union which was treated by bone grafting ${ }^{[4]}$.

Korani et al. found that maximum incidence was seen in the age group of 21 - 30 years $(38.88 \%)$. $83.33 \%$ cases were males with male:female ratio of $5: 1.77 .77 \%$ cases had Type I (extension type) injury. 22.23\% cases were of Type II (flexion). There were no cases of type III and Type IV fracture dislocation. $11.12 \%$ patients had compound fracture. $56.55 \%$ patients sustained injuries as a result of direct injury [10].

Reddy and Prasad reported that majority of the study participants were in the age group of 21-30years (45.16\%). Most of the study participants were males in $(61.2 \%)$. The fractures on the left side (71\%) were more common than those on the right side (29\%). Road traffic accidents were the cause of the fracture in most of the case (45\%), followed by fall on the outstretched hand $(39 \%)$ and violence $(16 \%)$. Most of the patients belonged to the Type I category $(67.8 \%)$ as Bado's classification. Equal incidence was found in Type II and III ( $16.1 \%$ each) and nobody was classified under Type IV. More than half of the cases were treated with DC plating. The outcome of the operation was excellent in $61.3 \%$, good results in $19.4 \%$ and fair outcome was seen in $16.6 \%$ while poor results were seen only in 1 case $(3.2 \%)^{[11]}$.

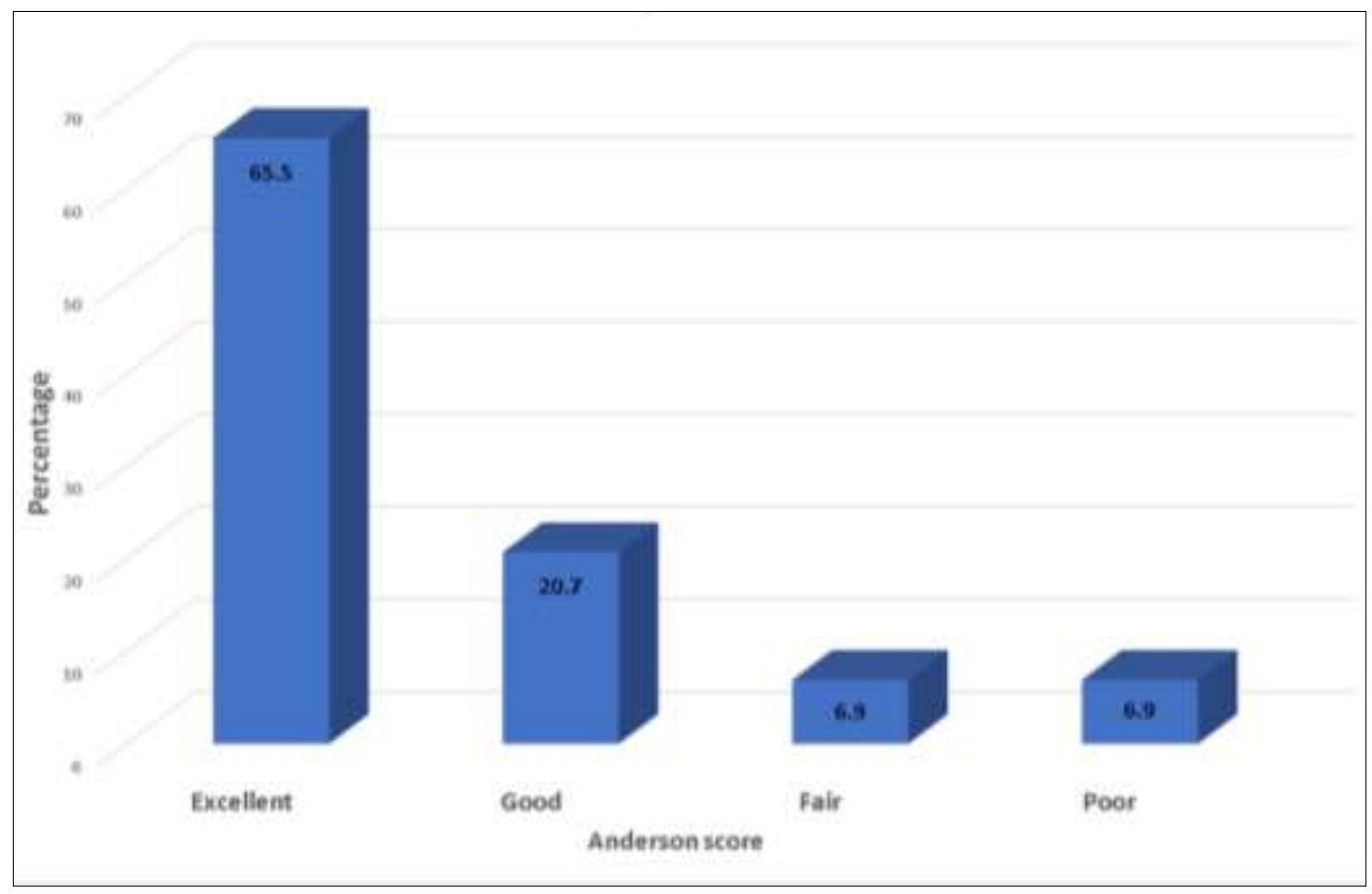

Chart 1: Showing functional outcome 


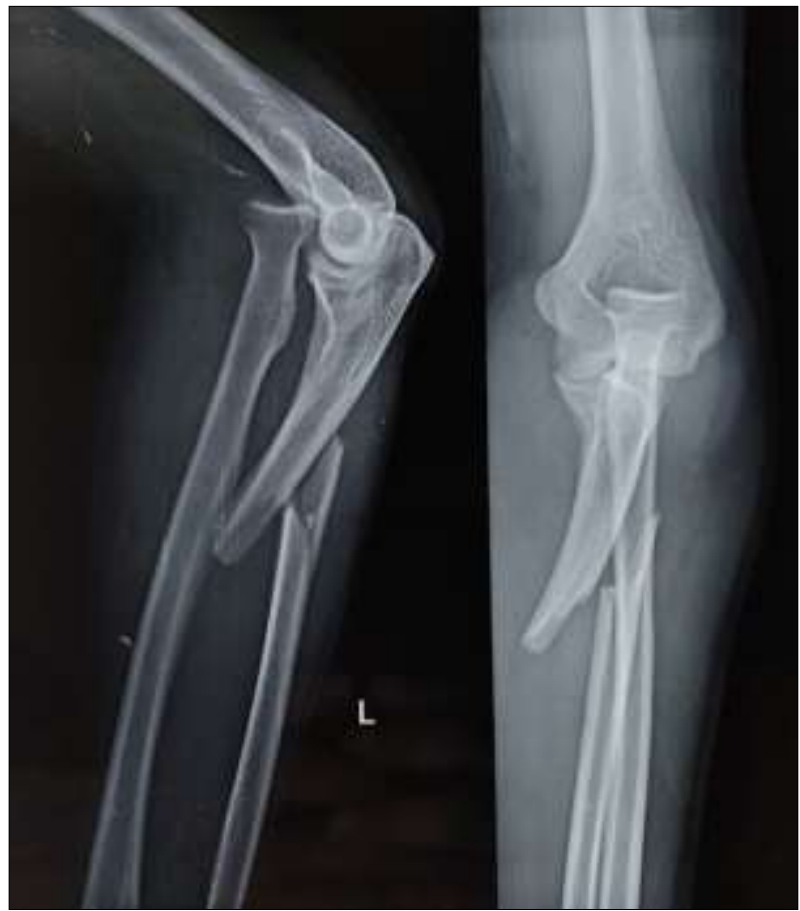

Fig 1: Showing pre-operative X-ray of Monteggia fracture dislocation

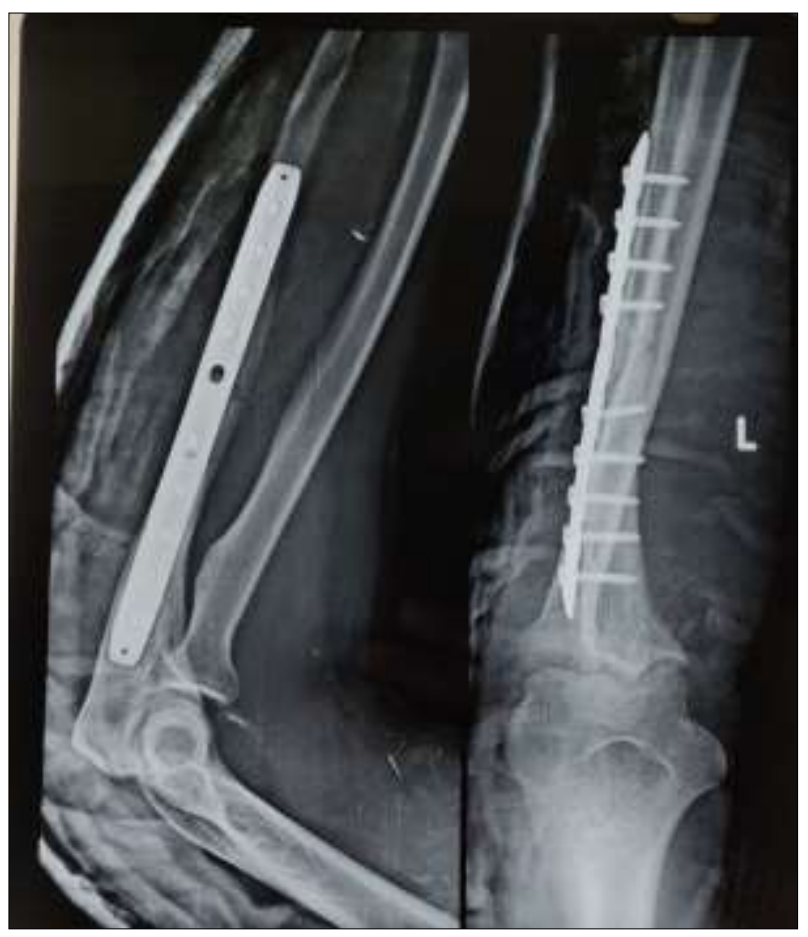

Fig 2: Showing post-operative X-ray

\section{Conclusion}

In the present study, excellent outcome was seen in $65.5 \%$ cases while poor result was obtained in $6.9 \%$ cases. Superficial infection was seen in three cases and non-union in two cases. Patients must be informed about potential functional deficits and the possible need for further surgery. Rehabilitation of the upper limb should be done as soon as possible to prevent elbow stiffness when the stability of the elbow is achieved.

\section{References}

1. Sajjan SS, Hegde AS, Annappa R, Mallya S. Monteggia type 1 fracture equivalent with concomitant ipsilateral distal radius and ulna fracture in an adult: A rare case report. Journal of Clinical and Diagnostic Research
2017;11(10):RD06-7.

2. Najd Mazhar F, Jafari D, Shariatzadeh H, Dehghani Nazhvani H, Taghavi R. Surgical Outcome of Neglected Monteggia Fracture-Dislocation in Pediatric Patients: A Case Series. Journal of Research in Orthopedic Science 2019;6(1):e83225.

3. Singh J, Kalia A, Dahuja A. Ipsilateral Radial Head Dislocation And Proximal One-Third Radial Shaft Fracture In An Adult: A Case Report. The Open Orthopaedics Journal 2018;12(1):189-95.

4. Khan SA. Monteggia fracture dislocation in adults: study of functional outcome following surgical treatment in patients attending a tertiary care center in North India. International Journal of Research in Medical Sciences 2017;5(7):2975-81.

5. Jepegnanam TS. Salvage of the radial head in chronic adult Monteggia fractures: Report of Four Cases. The Journal of Bone and Joint Surgery British 2006;88$\mathrm{B}(5): 645-8$.

6. Karkhur Y, Tiwari A, Bhatnagar N. Monteggia Fracture Dislocation with Ipsilateral Distal Radius - Ulna Fracture in a Child. Journal of Trauma and Orthopaedics 2017;12(2):23-6.

7. Agarwal A, Pawar I. Neglected Type I Monteggia Fracture Dislocation in Adult. J Clin Diagn Res 2017;11(8):RD01-2.

8. Papaioannou I, Repantis T, Baikousis A, Korovessis P. Adult Monteggia Lesion with Ipsilateral Distal Radius Fracture: A Case Report and Review of the Literature. J Orthop Case Rep 2018;8(3):77-80.

9. Babu P, Laalasa K, Kumar K, Venkappa C, Chandan S. Clinical Study Of Surgical Management Of Monteggia Fracture Dislocation In Adults, IJAR - Indian Journal of Applied Research(IJAR), IJAR/World Wide Journals Indian Journal of Applied Research 10(6):1-2.

10. Korani R. Monteggia fracture dislocation: A study on the functional outcome of surgical treatment in patients attending Osmania General Hospital, Hyderabad, Telangana, India. International Journal of Orthopaedics Sciences 2018;4(1):5-11.

11. Reddy GR, Prasad PN. A study to assess epidemiological, clinical profile and outcome of Monteggia fracture dislocation in adults: a retrospective study. Int J Res Orthop 2017;3(3):472. 